\title{
LUNG NODULES SEGMENTATION IN CHEST CT BY LEVEL SETS
}

\section{APPROACH}

\author{
Archana $\mathbf{A}^{\mathbf{1}}$, Amutha $\mathbf{S}^{\mathbf{2}}$ \\ ${ }^{1}$ Student, Dept. of CNE (CSE), DSCE, Bangalore, India \\ ${ }^{2}$ Professor, Dept. of CSE, DSCE, Bangalore, India
}

\begin{abstract}
Segmenting Lung nodules from a chest CT image have vital interest for medical applications like diagnosis and surgical planning. Here the focus is to segment the small lung nodules from lung CT scan by level set approach that uses signed distance function. A general lung nodule shape model is proposed using implicit spaces as a signed distance function which is fused with the image intensity statistical information. Mapping of shape model to image domain is done by a global transformation (inhomogeneous scales, rotation, and translation) and is matched with the image implicit representations to handle the alignment process. Shape alignment process is handled by evolving transformation parameters through gradient descent optimization that marks the boundaries of the nodule. Image intensity as well as prior shape information is used overlay images to segment out the nodule. A nonparametric density estimation approach is employed to handle the Statistical intensity and background region. This algorithm aims to segment the nodule irrespective of its type or location.
\end{abstract}

\section{INTRODUCTION}

Lung cancer is a deadly disease in the world. The early detection of lung cancer has a vital role in medical science. The segmentation of nodule and its classification has healthy and cancerous is challenging. A computerized method of diagnosis commonly referred as computerassisted diagnosis (CAD) for various imaging modalities using computer vision and machine learning methods. Radiologist use the intensity, shape and texture information to differentiate the change in image intensity or Hounsfield Units (HU) of normal tissues and abnormalities while reading the chest $\mathrm{CT}$. Thus, they differentiate health tissue and abnormalities or nodules.

CAD models are built to enhance the image quality which would help radiologist for diagnosis as well for the early detection of lung cancer. Since Lung cancer is a leading cause of death [1], best chances of survival comes with the early detection of cancer. CAD system detects nodules, and then categorization of nodule and segmentation is carried out. It is important area in biomedical image analysis in which computer vision, image processing, visualization, and machine learning approaches have their role in development and validation of these systems. For object segmentation lung nodules are considered as objects. Real world objects' modeling is difficult hence segmentation process is never an easy task. And in case of lung nodules it is even more challenging cause of size constraints when nodule is less than $1 \mathrm{~cm}$. Segmentation allows radiologists to measure and follow up lung nodules size which is one of the most reliable indicators of malignancy of some nodule types.

CT imaging technology has evolved rapidly over the past decade, first with the move toward thin-slice high-resolution CT and helical CT scanning, and then with a push toward multi-detector row $\mathrm{CT}$ that allows multiple projections to be acquired simultaneously. These technological advances have led to improved resolution, reduced scan times, and overall, a vast improvement in the quality of the image data. Along with improvement in hardware, there is a growing interest in computerized analysis of the image data. It is been noted that the area of pulmonary image processing for CT images alone has been increasing at a pace of about $50 \%$ per year for the past five years. This growth rate shows pulmonary imaging to be an exciting research area in which to work.

The lung is the essential respiration organ in many airbreathing animals. In mammals and other more complex life forms, the two lungs are located near the backbone on either side of the heart. Their primary function is to transport oxygen from atmosphere into the bloodstream, and to release carbon dioxide from bloodstream into the atmosphere. Since the oxygen needed for entire body is supplied by lung, a large area is needed for this exchange of gases which is accomplished by the mosaic of special groups of cells with tiny, exceptionally thin-walled air sacs known as alveoli. The functioning of respiratory system is discussed below with the aid of anatomical visualization. Lungs are a complex organ; it includes several structures (anatomical objects), such as vessels, fissures, bronchi or pleura that can be located close to lung nodules. Hence lung nodules segmentation in CT imaging is a complex and challenging process. Also, the main "head" of the nodule is what radiologists consider when computing the size. In the case of detached nodules it is referred as well-circumscribed nodules the whole segmented nodule is considered in size computations and growth analysis. The attached nodules are grouped as juxta-pleural, vascularised and pleural-tail the "head" is required to be extracted from the anatomical surrounds. The "head" in case of lung nodule is shortly explained. 

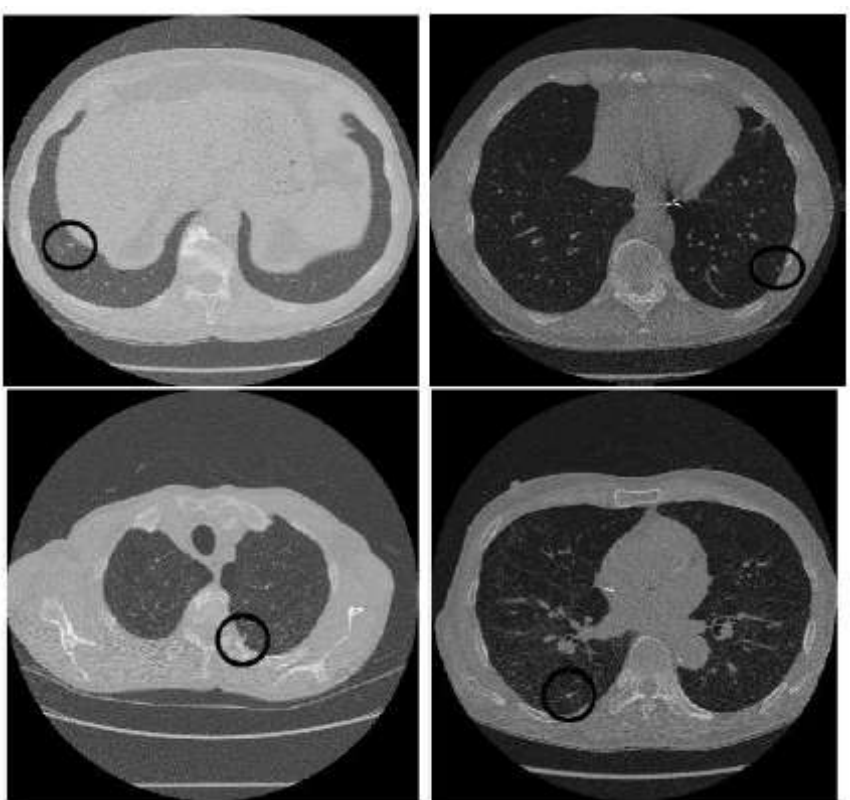

Fig 1. Four examples of CT images that contain nodules: (a) Well- Circumscribed, (b) Pleural-Tail, (c) Juxta-Pleural, and (d) Vascularized.

In figure above a black circle is drawn around the nodule center to indicate the nodule regionand that would be the region of interest for the purpose of segmentation.

Despite the wide range of nodule classifications among radiologists, the nodule classification of Kostis et al. [2]. Nodules in Kostis's work are grouped into four main categories:

i) Well-circumscribed where the nodule is located centrally in the lung without being connected to vasculature.

ii) Vascularized where the nodule has significant connection(s) to the neighboring vessels while located centrally in the lung

iii) Pleural tail where the nodule is near the pleural surface, connected by a thin structure.

iv) Juxta-pleural where a significant portion of the nodule is connected to the pleural surface.

In the juxta-pleural case the main shape information attached to the pleural-wall is the "head" of the nodule and is used in size and growth follow-up computations which can aid in deciding malignancy. The idea behind segmentation of this type of nodules is that the lung nodules do not share common shape information but in all instances the main nodule "head" can be contained within an elliptic region.. The circular/elleptic model is implicitly represented by a signed distance function and combined with the nodule image information to give the final results. The combination is formulated as a shape registration process by matching implicit space. The nodule shape model is registered with the image information by employing a transformation with inhomogeneous scales, rotation, and translation. The gradient descent optimization is used to estimate the transformation parameters. The framework supports embedding prior nodules shape information to overcome challenges of the segmentation process such as noise, in- homogeneities, and cluttered data. The challenge with dimensionality representation of the shapes by the implicit signed distance representation is addressed thoroughly in the level set segmentation literature using a narrow band implementation. In the context of shape registration, one can reduce the sample domain for registration to a narrow band around the input shape in the embedding space. More sophisticated nodule models maybe used instead of the elliptical which are sensitive to the specific nodule category. The segmentation approach would be applied in parallel with the nodule model per category applied followed by a decision step. Small-size nodules that are $1 \mathrm{~cm}$ or less in diameter, nodule topologies are harder to discriminate; another reason an elliptical nodule model to segment the nodule's "head" region is sufficient. The technique is effectively segment nodule with diameters varying from 2 $\mathrm{mm}$ to $20 \mathrm{~mm}$, whereas existing techniques are applied on nodules $>1 \mathrm{~cm}$.

\subsection{Problem and Motivation}

Many research projects are proposed and being implemented since several years, to efficiently screen lung cancer with low dose CT in asymptomatic people at high risk. The feature for the characterization of indeterminate small (nodule less than $1 \mathrm{~cm}$ ) screening detected lung nodules is the growth of size. Nodule size is an important factor in volumetric analysis of lung nodules. Size of the nodule is linked to nodule malignancy, with non-calcified nodules greater than $2 \mathrm{~cm}$ in diameter having a higher rate of malignancy than smaller nodules. Size computation through a 3D measurement is usually performed by applying volumetric methods to a segmentation result. Segmentation of nodules of smaller diameter, suppressing anatomical structures in lungs is challenging. Variational level set approach is used to model the nodule image information. Intensity distribution is modeled as a nonparametric Gaussian Kernel-Based estimation. Object and background probability density function are adaptive and they are estimated at each iteration of level set solution with the gradient descent optimization.

Prior shape model is considered for lung nodules. Circle model is a shape prior represented by a signed distance function since the "head" is contained with head region.

The circular model is implicitly represented by a signed distance function and combined with the nodule image information to give the final results.

Amal Farag, James Graham and Aly Farag in [3] aimed at automatic detection and classification of nodules from LDCT scanning; a step for early diagnosis of lung cancer. This is an approach for segmentation based on combination of EM algorithm and morphological operations. They compared this with two other approaches that are based on level sets and energy optimization by the Graph Cuts technique. As always, with histogram-based approaches, some region interferences is deemed to happen, especially as the boundaries are usually ragged and contains various areas of similar gray scale distributions. Morphological dilation 
using a circular structuring element is then applied as a smoothing filter on the contour of the segmented lung region in order to avoid losing nodules which are attached to the lung walls. To decrease the sensitivity of the segmentation result to the structuring element diameter, apply it to the inner and outer lung region contour. This approach, in practice is used 2D analysis is due to the limitations of the LDCT scans in terms of resolution and the fact that the nodules of interest are within a spatial support. The algorithm flow is like this, Slices are loaded serially and obtain the gray level histogram then EM algorithm is applied to obtain a fitting for the PDF function representing the histogram, and obtain an optimum segmentation threshold for the lung and the rest. Boundary regions are enhanced by successive morphological operators.

\subsection{Level Sets Method}

Object region in image covered by a boundary region. Region boundaries are called the shape of the region or object. If this object can be represent any real time geometric shape, then it can be used for shape analysis. Choosing out such geometric shape for object region is key step in several medical and computer vision applications such as registration and segmentation [4]. The level set function as a signed distance map is able to capture complicated topology and deformations. The object of interest is represented by a contour defined implicitly as a level set function. For this contour, some initialization has to be done which evolves according to a partial differential equation for the final object solution. The formulation of the intensity segmentation process in order is as follows:

Let $\mathrm{Q}=\{0, \ldots Q-1\}$ denote a set of gray levels $\mathrm{q}$ where $\mathrm{Q}$ is the number of gray levels.

Let $\Omega=\left\{\mathrm{X}=[\mathrm{x}, \mathrm{y}]^{\mathrm{T}}: 0 \leq x \leq X, 0 \leq y \leq Y\right\}$ be a finite domain for the image of interest $I: \Omega \subset \mathrm{R}^{2} \rightarrow \mathrm{Q}$. And $f: \mathrm{Q} \rightarrow \mathrm{R}$ is an empirical frequency distribution of gray levels $q$ collected over the whole image I such that $\Sigma_{\mathrm{q}=0}^{\mathrm{Q}} \mathrm{f}(\mathrm{q})=1$. The segmentation process aims to partition the image into two regions: Object (inside the contour denoted by $\mathrm{O}$ ) and Background (outside the contour denoted by B).

Level set function $\phi: \Omega \subset \mathrm{R}^{2} \rightarrow R$ is defined as minimum Euclidean distance between the point $X \in \Omega$ and the shape boundary and curve represents the shape boundaries of a certain shape. The distance takes a negative sign outside the shape boundaries. Curve is initialized inside an object, and it evolves to cover the region guided by image information. The evolving curve within the level set formulation is a propagating front embedded as the zero level of a 3D scalar function, $\phi(\mathrm{X}, t)$. Contour representation is used to formulate intensity segmentation. Error can be computed by counting the number of correctly classified pixels and then measuring the difference with respect to the total number of pixels. This is done by calculating the summation of the probabilities of the internal pixels as object and the external pixels probabilities as background. This is measured by the term:

$$
\text { Error }=1-\pi_{o} \int_{\Omega_{-}} P_{o}(X) d \Omega-\pi_{B} \int_{\Omega_{+}} P_{B}(X) d \Omega
$$

Here, and are the region outside and inside the object, respectively.

Accounting to the intensity values $P_{\sigma}(\mathrm{X})=P(I(\mathrm{X}) \mid O)$ and $P_{B}(\mathrm{X})=P(I(\mathrm{X}) \mid B)$ would be the probability density functions for the object and background respectively.

Prior probabilities of regions $\left(\pi_{0}\right.$ and $\left.\pi_{\mathrm{B}}\right)$ are used in the formulation $\left(\pi_{0}+\pi_{\mathrm{B}}=1\right)$.

$$
p(q)=\pi_{o} p(q \mid O)+\pi_{B} p(q \mid B)
$$

Energy minimization is done using,

$$
\begin{aligned}
E(\phi)= & \pi_{o} \int_{\Omega} p(I(X) \mid O) H_{\in}(\varphi) d \Omega \\
& -\pi_{B} \int_{\Omega} p(I(X) \mid B)\left(1-H_{\in}(\varphi)\right) d \Omega
\end{aligned}
$$

where $\mathrm{H}$ is the Heaviside step function and $\epsilon$ is a positive numerical value representing the narrow band region width. An extra term is added to the energy function which represents the contour arc-length $\left(\mathrm{L}=\int_{\Omega} \delta_{\in}(\phi)|\nabla \phi| d \Omega\right)$. The minimum arc-length guaranties smooth evolution. The new energy will be:

$$
\begin{aligned}
E(\phi)= & \pi_{o} \int_{\Omega} p(I \mid O) H_{E}(\varphi) d \Omega \\
& \quad-\pi_{B} \int_{\Omega} p(I \mid B)\left(1-H_{\in}(\varphi)\right) d \Omega+L \lambda
\end{aligned}
$$

where $\lambda \in \mathrm{R}+$. Another term can be added to the energy function in order to grantee that the level set evolves as a signed distance function with a gradient magnitude equal to one, represented as $\frac{1}{2}(|\nabla \phi|-1)^{2} \mid$ and blended by the positive real argument $\mu$. The final energy will be as follows:

$$
\begin{aligned}
& E(\phi)=\pi_{o} \int_{\Omega} p(I \mid O) H_{\epsilon}(\varphi) d \Omega \\
& -\pi_{B} \int_{\Omega} p(I \mid B)\left(1-H_{\epsilon}(\varphi)\right) d \Omega \\
& +\lambda \int_{\Omega} \delta_{\epsilon}(\phi)|\nabla \phi| d \Omega+\int_{\Omega} \frac{1}{2}(|\nabla \phi|-1)^{2} d \Omega
\end{aligned}
$$

The level set function evolves to minimize such functional using the Euler-Lagrange formulation with the gradient descent optimization:

$$
\frac{\partial \phi}{\partial t}=\delta_{\epsilon}(\phi)\left(\pi_{o} p(I \mid O)-\pi_{o} p(I \mid B)\right)+\lambda k+\mu(\Delta \phi-k)
$$


where $\delta$ is the derivative of the Heaviside function and $\kappa$ is the curvature. Appendix contains detailed derivation of Eq. 6. We note that the evolution depends on the local geometric properties (local curvature) of the front and the external parameters related to the input data I . The function $\phi$ deforms iteratively according to above equation, while solving $\phi(X, t)=0$ gives the position of the 2D front at each iteration. The prior probabilities are proportional to the number of pixels in the object and background respectively as follows:

$$
\pi_{o}=\int_{\Omega} H_{\epsilon}(\bar{\phi}) d \Omega / \int_{\Omega} d \Omega
$$

The probability density functions in the energy functional are approximated using a nonparametric Gaussian KernelBased Density Estimation model.

\subsection{Prior Shape Model}

The shape prior model is aimed to compute a transformation of object that moves a source shape $(\alpha)$ to its target $(\beta)$ [5], [6]. Assume that the source and target shapes are represented by the signed distance functions $\phi \alpha$ and $\phi \beta$ respectively.

The transformation function is assumed to have scaling components; $\mathrm{S}=\operatorname{diag}\left(\mathrm{S}_{\mathrm{x}}, \mathrm{S}_{\mathrm{y}}\right)$, rotation angle; $\theta$ (associated with a rotation matrix $R$ ) and translations; $T=\operatorname{transpose}\left[\mathrm{T}_{\mathrm{x}}\right.$, $\mathrm{T}_{\mathrm{y}}$ ] an energy function can be obtained where $\mathrm{E} \leq \mathrm{E} 1$

$$
E=\int_{\Omega} \delta^{\prime}\left(\phi_{\alpha}, \phi_{\beta}\right)\left(s \phi_{\alpha}(X)-\phi_{\beta}(A)\right)^{2} d \Omega
$$

Representing the shapes as a signed distance transform has several benefits such as the registration energy functional being rotation and translation invariant, further enhancement in the proposed approach is incorporation of a scaled version of the source shape representation which does not require point correspondence since Eulerian framework is used.

The above described energy function may be optimized using various approaches such as the Gradient Descent such as the Levenberg, Quasi-Newton, Levenberg-Marquardt. The Gradient Descent approach is used to carry out the optimization in this paper since the signed distance satisfies a condition for the convergence of the gradient descent method [7]. The required functions involved have to be differentiable, thus, a smeared version of the function, $s\left(S_{x}\right.$, $\left.S_{y}\right)=\max \left(S_{x}, S_{y}\right)$ is used at the line $\left(S_{x}=S_{y}\right)$ since the function is not differentiable here, that is based on its original definition: $s\left(S_{x}, S_{y}\right)=\max \left(S_{x}, S_{y}\right)=S_{x} H_{\in}\left(S_{x}-S_{y}\right)$ $+S_{y}\left(1-H_{\epsilon}\left(S_{x}-S_{y}\right)\right)$, which will return $S_{x}$ if $\left(S_{x}-S_{y}\right) \geq 0$, otherwise $S_{\mathrm{y}}$.

The smeared Heaviside step function $\mathrm{H}$ is used to obtain a smooth transition around the line $S_{x}=S_{y}$ allowing the function to be differentiable everywhere. The function derivatives will be calculated as:

$$
\begin{gathered}
\partial_{s} \partial_{s x}=H_{\in}\left(s_{x}-s_{y}\right)+\left(s_{x}-s_{y}\right) \delta_{\in}\left(s_{x}-s_{y}\right) \text { and } \\
\partial_{s} / \partial_{s y}=H_{\in}\left(s_{y}-s_{x}\right)+\left(s_{y}-s_{x}\right) \delta_{\in}\left(s_{y}-s_{x}\right) .
\end{gathered}
$$

The parameters $\left\{\mathrm{S}_{\mathrm{x}}, \mathrm{S}_{\mathrm{y}}, \theta, \mathrm{t}_{\mathrm{x}}, \mathrm{t}_{\mathrm{y}}\right\}$ are required to minimize the energy functional $\mathrm{E}$. The gradient descent optimization process is controlled by the following equations:

$$
\begin{gathered}
\frac{\partial a}{\partial t}=\int_{\Omega} \delta^{\prime} r^{\prime}\left(\frac{\partial S}{\partial a} \phi_{a}(X)-\left(\nabla \phi_{\beta}(A)\right)^{T} \nabla_{a} A\right) d \Omega \\
\left.\frac{\partial b}{\partial t}=\int_{\Omega} \delta^{\prime} r^{\prime}\left(\nabla \phi_{\beta}(A)\right)^{T} \nabla_{b} A\right) d \Omega
\end{gathered}
$$

Where $r^{\prime}=s \phi_{a}(X)-\phi_{\beta}(A), a \in\left\{s_{x}, s_{y}\right\}$, and $b \in\left\{\theta, t_{x,}, t_{t}\right\}$.

\section{METHOD OF IMPLEMENTATION}

The algorithm can be divided into three main tasks:

1. Pre-processing which consists of several key steps that leads to having a cropped region of interest centred around on the nodule.

2. Computation of the initialization parameters used for the segmentation approach and generation of the signed distance representation of the shape model, and

3. Perform the shape based level set algorithm where correct convergence occurs when the nodule "head" is completely extracted. The steps are described in more details below.

\subsection{Preprocessing}

This step is to segment out region of interest from chest CT scan image. The lung tissue is extracted from surrounding pleural surface statistical intensity based approach [5]. This eliminates chest cavity and other high intensity anatomical objects from the chest CT image leaving behind the lung tissue. Lung nodule detection is performed to obtain candidate nodule locations. Candidate nodule location is found and a post processing stage is carried out. Post processing provides the cropped regions of interest of nodule candidates. A box is set around a nodule centre and the surrounding neighbour is extracted. The image resulted (small image containing region of interest) will be the input for segmentation steps. The ROI for nodules $\leq 1 \mathrm{~cm}$, for example, was empirically shown to be within a small range of pixels region. 


\subsection{Initializing Contour and Transform function}

First the initial probability distributions of the object and background intensities are computed as Gaussian functions using the expectation maximization (EM) algorithm. This information is used to mark the object and background regions. Based on this, the signed distance function is computed as $\phi g$ [6]. Initialize $\lambda$ and $\mu$ and compute the prior probabilities and the probability density functions for the object and background for each intensity levels is solved to compute the intensity segmentation region implicitly represented by $\phi g$. The computations are repeated iteratively until $\phi g$ reaches saturation level. If it does not saturate to a level of minimum change, repeat the steps. For prior shape model, initialize the transformation parameters to $\mathrm{s}_{\mathrm{x}}=1, \mathrm{~s}_{\mathrm{y}}=$ $1, \theta=0$. (Arbitrary values chosen).

\subsection{Segmentation}

This process aims to segment out the lung nodule from the background. Construct the initial prior shape model and corresponding signed distance representation, $\phi$ p. Elliptical model is created using the nodule image size information. In the test image, nodule center is marked to initialize the values for the translation parameters. Automatic seed-point placement can also be used to position the nodule within an image. Later, the gradient descent approach is solved to the energy. Parameters converge to their steady state values and hence, the final boundaries of the ellipse are computed. The final nodule boundary is marked by solving: $\phi \mathrm{p}$ and $\phi \mathrm{g}$ that is, overlaying image obtained with prior shape model with image obtained by signed distance function, considering the points satisfying the conditions $\phi \mathrm{p}(\mathrm{X}) \geq 0$ and $\phi \mathrm{g}(\mathrm{A}(\mathrm{X})) \geq 0$

\section{RESULTS}

The algorithm is validated for the images obtained from ELCAP public database, (DB1), the database consists of 50 sets of LDCT lung scans $(30-40 \mathrm{~mA})$ taken at a single breath-hold, with slice thickness $1.25 \mathrm{~mm}$ and resolution 0.5 $\mathrm{mm} \times 0.5 \mathrm{~mm}$. Locations of 397 nodules, provided by radiologists, where used to create a database that consists of $39.12 \%$ juxta-pleural nodules, $13.95 \%$ vascularised nodules, $31.29 \%$ well-circumscribed nodules and $15.65 \%$ pleural-tail nodules. A subset database containing 291 nodules is used. This database has nodules of diameter ranging from $2 \mathrm{~mm}$ to $5 \mathrm{~mm}$. This approach uses a region of interest (ROI) image that contains the lung nodule in the center as input. Expectation Maximization algorithm is used initially to estimate the probability density functions for the lung and non-lung tissues in the ROI image. The probability density functions are assumed to be Gaussian and it is used to initialize the level set function $\phi \mathrm{g}$ by computing the signed distance transform of the resulting binary image.

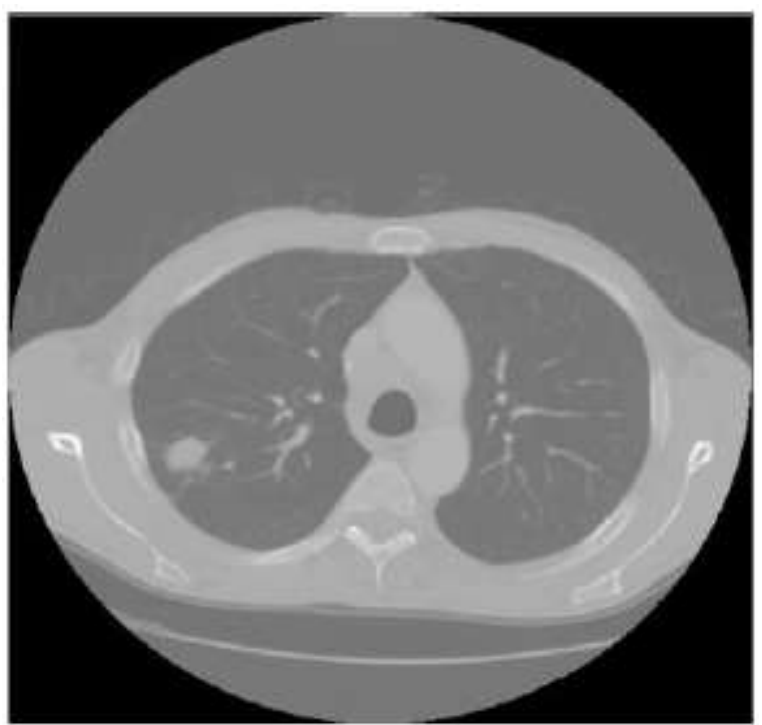

Fig 2. Input chest CT image (ELCAP Lung Database)

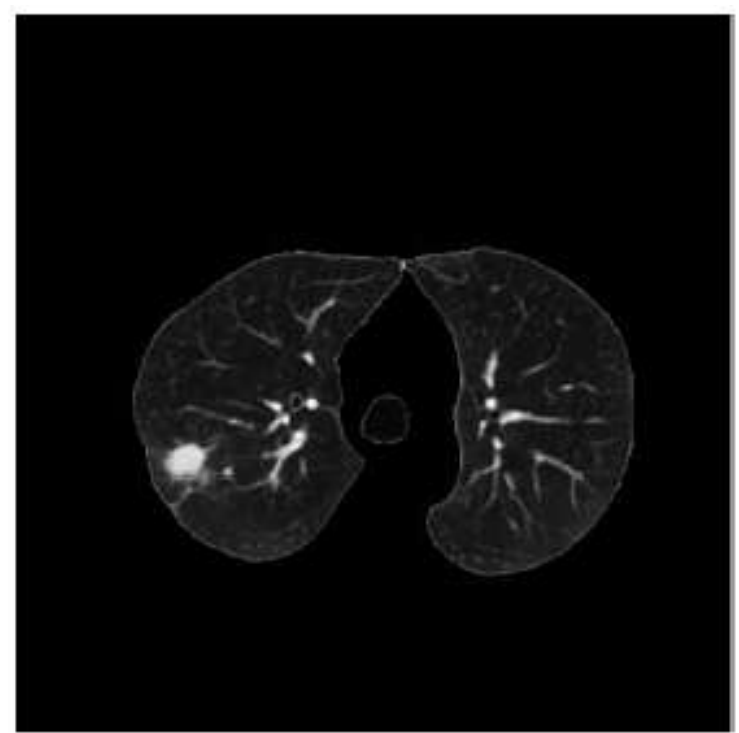

Fig 3. Lung tissue extracted from the chest CT image

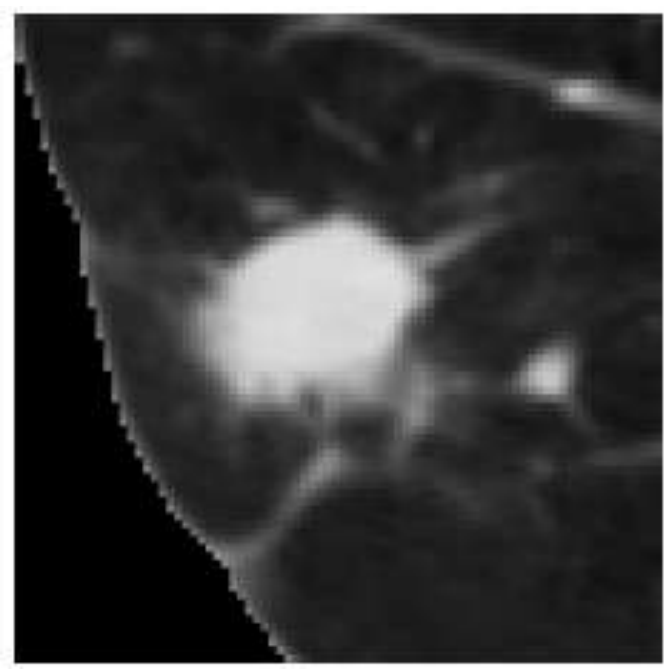

Fig 4 . ROI obtained by preprocessing 


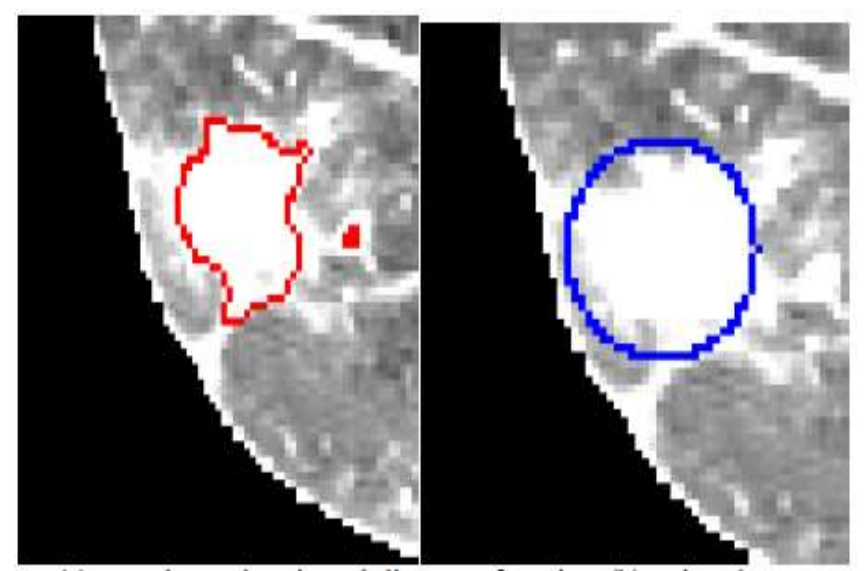

Fig 5. (a) Level Sets by signed distance function (b) Prior shape model applied on ROI

The level set function evolves iteratively to cover the object of interest for a pre-processed image, the prior probabilities and the probability density functions of the object and background are updated and computed.

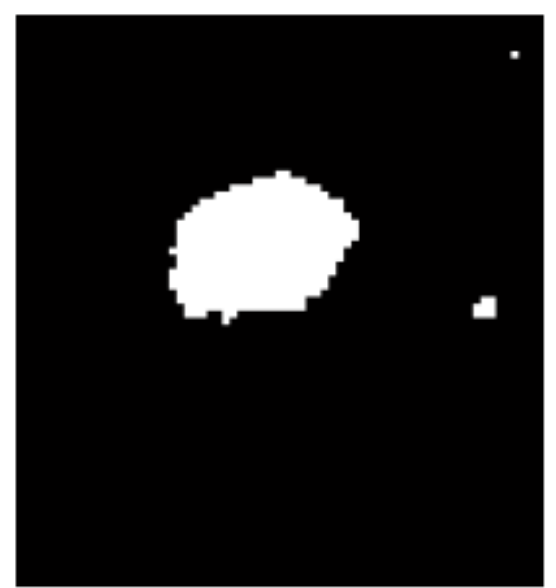

Fig 6 Resulting image after combining images obtained by level sets and prior model

By this approach, inhomogeneities in either the lung or nonlung tissues can be easily detected where as the conventional EM algorithm thresholding technique fails to deal with this issue. These results are compared with the nodule identified by the radiologists. It showed a good performance: a TP rate of $95 \%$ is achieved combined with $10 \%$ FP. The results show accuracy of $81 \%$ when having $1 \%$ FP rate.

\section{CONCLUSION}

The algorithm proposed for Variational formulation for the lung nodules segmentation problem using level sets. A circle prior shape model is embedded into the image domain by means of registration which allows the use of inhomogeneous scales. The use of inhomogeneous scales in registering the shape and intensity models allow the template to deform as an adaptive ellipse to exactly fit the nodule boundaries. The registration process incorporates prior shape model as well as image intensity information in implicit spaces. The resulting nodule sizes across the scans give a possible indication about nodule(s) malignancy.

\section{REFERENCES}

[1]. American Lung Association (2012). Lung Cancer Fact Sheet, Washington, USA[Online]. Available: http://www.cancer.gov.

[2]. W. J. Kostis, A. P. Reeves, D. F. Yankelevitz, and C. I. Henschke, "Three dimensional segmentation and growthrate estimation of small pulmonary nodules in helical CT images," IEEE Trans. Med. Imaging, vol. 22, no. 10, pp. 1259-1274, Oct. 2003.

[3]. Amal Farag, James Graham and Aly Farag in "Robust Segmentation of Lung tissue in chest CT scanning", 2010.

[4]. K. Siddiqi, A. Shokoufandeh, S. Dickinson, and S. Zucker, Shocks, graphs and shape matching," IEEE, Jan. 1999.

[5]. H. Abdelmunium and A. A. Farag, "Curve/surface representation and evolution using vector level sets with application to the shape-based segmentation problem," IEEE Jun. 2007.

[6]. H. Abdelmunim, A. Farag, W. Miller, and M. AboelGhar, "A new CAD system for the evaluation of renal rejection using DCE-MRI," in Proc. IEEE Comput. Soc. Workshop MMBIA, Jun. 2008, 